\title{
The Improved TBW Versus the Standard TBW for Olecranon Fractures in the Aged: a Retrospectively Comparative Study
}

Xiaoyong Lin ( $\sim 471933149 @ q q . c o m$ )

Ruian City People's Hospital

Hai Liao

Ruian City People's Hospital

Research article

Keywords: Olecranon fracture, Tension band wiring, Comparison Introductiontures

Posted Date: June 14th, 2021

DOI: https://doi.org/10.21203/rs.3.rs-549448/v1

License: (c) (1) This work is licensed under a Creative Commons Attribution 4.0 International License.

Read Full License 


\section{Abstract}

Objectivession: Olecranon fractures of the aged are traditionally managed operatively with Tension Band Wire (TBW). We compared clinical outcomes of treatment of the improved TBW versus the standard TBW for the treatment of the senile Olecranon fractures patients.

Methods: A retrospective study was conducted on senile olecranon fractures patients in our hospital from June 2016 to January 2019. After appropriate exclusion, 62 olecranon fractures patients (29 patients in group A of the improved TBW, 33 patients in group B of the standard TBW) were reviewed in this study. All reviewed patients underwent preoperative immobilization and detumescence, open reduction and internal fixation, and postoperative function exercise. Duration of surgery, intraoperative blood loss, times of fluoroscopy intraoperatively, postoperative pain score, fracture union time, soft tissue irritation, failure of fixation and Broberg Morrey score of elbow function were recorded and compared.

Results: The duration of surgery, intraoperative blood loss, postoperative pain score, Broberg Morrey score of elbow function had no significant difference between the two groups. The improved TBW (group A) was better than the standard (group B) in terms of the fixation loosening, fracture union time, the skin irritation, and the difference was significant ( all $p<0.05$ ) .

Conclusions: On the basis of no additional surgical trauma, the improved TBW was not only benefit for the fracture union, but also benefit for reducing the incidence of internal fixation loosening and soft tissue irritation. This improved internal fixation is not an unattractive option for senile olecranon fractures patients.

\section{Introduction}

With an increasingly aging population, olecranon fractures in the elderly are likely to be encountered at higher frequency[1]. Olecranon fractures are among the common injuries sustained by the elderly that account for about $10 \%$ of all upper limb fractures of the elderly[2]. Tension band wiring (TBW) has been widely used as a method of reliable fixation for Olecranon fractures, particularly for the elderly. TBW not only reduces the incidence of traumatic arthritis, but also achieves satisfactory elbow joint function[3]. Besides,TBW has the advantages of simple operation and less trauma. But at the same time, it has complications such as internal fixation failure and soft tissue[4], which affects clinical efficacy. The improved TBW which uses the perforated Kirschner Wire instead of the standard Kirschner Wire, is a further improved internal fixation on the basis of the standard TBW technique. At present, the application and comparative study of perforated Kirschner wire in olecranon fracture are less, so our medical team used a retrospective case-control study to explore the clinical efficacy of the improved TBW, so as to provide reference for the selection of internal fixation.

\section{Data And Methods}

\section{Patients}


The approval for the study was obtained from our hospital's Review Board and informed consent from enrolled patients. Data before hospitalization and continued forward until the latest office visit postoperatively were recorded.A total of 62 old patients with olecranon fracture and a mean age of $70.7 \pm$ 16.8 years old (range, 60-90 years) who were treated in authors' Institutional from June 2016 to January 2019 , were included in the study according to inclusion and exclusion criteria.

Inclusion criteria: 1.ages from 60 to 90 years old; 2 .new and closed fracture without an open elbow wound; 3. Mayo I and II fracture; 4. internal fixation of the improved or standard TBW. Exclusion criteria: 1. comminuted fracture, coronoid process and long oblique fracture; 2 . pathological fracture; 3 . other fractures that may have influence on elbow motion such as humerus fracture, olecranon fracture and so on; 4. neurovascular injury; 5 . injury of important organs; 6 . congenital or acquired deformity of the same side elbow joint; 7. lost to follow-up patients. According to the different internal fixation methods, the enrolled patients were classified into two groups. All cases were conducted by the same group of experienced elbow joint surgeons. The work had been reported in line with the STROCSS criteria.

Demographics and perioperative data were reviewed for each patient. Preoperative data included age, sex, fracture classification, BMI index. Perioperative data included duration from injury to surgery, duration of surgery, intraoperative blood loss, times of fluoroscopy intraoperatively, postoperative pain score, fracture union time, soft tissue irritation, failure of fixation and Broberg Morrey score of elbow function.

\section{Surgical procedure}

Made an incision about $10-16 \mathrm{~cm}$ long originated from humerus, extending across the olecranon, end the position of upper ulna. Fully exposed the fracture end, removed the blood clot and small bone fragments, decompressed the soft tissue from fracture crevice. The elbow joint was placed at $130^{\circ}$, then the fracture was reduced under direct vision until the local articular surface was restored to be flat. The reduction was maintained by a big clamp.

The improved TBW(group A): Two perforated K-Wire with diameter of $2 \mathrm{~mm}$ drilled parallel from the proximal of olecranon, then went along the medullary cavity and finally placed in the medullary cavity of ulna. About $3 \mathrm{~cm}$ from the distal end of the fracture position, an anchor hole with a diameter of $2 \mathrm{~mm}$ was drilled on the vertical long axis of the ulna. One steel wire or cable threaded into both the anchor hole and the Kirschner pin hole, then placed crossed at the back of the electron like Fig. 8. The hammering made the hole at the tail end of the Kirschner pin buried in the beginning of the triceps brachii muscle, tightened the Fig. 8 tension belt, and broke the part of the tail.

The standard TBW(group B): Two standard K-Wire with diameter of $2 \mathrm{~mm}$ was drilled through the fracture line parallel medullary cavity from the proximal part of the olecranon, placed in the medullary cavity of the ulna, and bent its tail. About $3 \mathrm{~cm}$ from the distal end of the fracture position, an anchor hole with a diameter of $2 \mathrm{~mm}$ was drilled on the vertical long axis of the ulna. One steel wire or cable threaded into 
the anchor hole, went bypass the tail bending part of the Kirschner wire, cross Fig. 8 at the back of the olecranon, and tighten the knot.

\section{Postoperative care, follow-up and therapeutic evaluatioan}

Regularly wound cleaning, passive functional exercise were made within 2 weeks, and active elbow joint functional exercise was gradually made after 2 weeks. The duration of surgery, intraoperative blood loss, times of fluoroscopy intraoperatively, postoperative pain score, fracture union time, failure of fixation, soft tissue irritation and Broberg Morrey score of elbow function were recorded and compared. The mean VAS score from the first day to the discharge date was used as the postoperative pain score of this study.Reviewed and evaluated the X-ray of the patients on the third day, the second week and every month after the operation. We observed whether complications such as fixation failure and soft tissue irritation appeared during follow up.And evaluated the bone union at the last follow-up, to decide whether to remove the internal fixation or not.At the final follow up, evaluated the degree of elbow degeneration according to the Broberg - Morrey classification[5]: the total score was 100 from the four aspects of exercise, strength, stability and pain. $95-100$ is excellent, $80-94$ is good, $60-79$ is $0 \mathrm{~K}, 0-59$ is poor.

\section{Statistical methods}

We use Statistical analysisarAdopt SPSS 20.0 software for data statistical analysis. Continuous data is presented with mean \pm standard deviation (SD), which were compared by t-test between 2 groups. Categorical data are expressed as percentage or rate compared by chi-square or $\chi 2$ test or fishertest.Statistical significance defined as $\mathrm{P}<0.05$.

\section{Result}

\section{Patient characteristics}

After screening and exclusion, a total of 62 patients were studied in this study from June 2016 to January 2019 , including 36 male cases and 26 female cases. The average age was $70.7 \pm 16.8$ years old (range, 60-90 years). All patients were followed up for $20.3 \pm 4.5$ months (range, $15-25$ months). The patients were divided into two groups according to different operation methods. There were 29 patients in group $\mathrm{A}$ (improved TBW) including 16 males and 13 females. The average age was $68.0 \pm 11.4$ years old, and the average BMI index was $26.4 \pm 5.2$. According to Mayo classification, there were 11 cases of type I and 18 cases of type II in group A; There were 33 patients in group B (the standard TBW) including 20 males and 13 females. The average age was $70.8 \pm 9.5$ years old, and the average BMI index was $27.1 \pm 6.4$. According to Mayo classification, there were 13 cases of type I and 20 cases of type II in group B.The average time from injury to surgery was $5.3 \pm 1.7$ days in group $A$ and $5.1 \pm 2.0$ days in group $B$. The demographic characteristics, such as age, sex, BMI index,time from injury to surgery and Mayo types, were similar between the groups ( $p>0.05$; table 1$)$.

\section{Perioperative data}


The duration of surgery, intraoperative blood loss, times of fluoroscopy intraoperatively, and postoperative pain score were as follows in group A: $82.6 \pm 23.9 \mathrm{~min}, 119.6 \pm 30.1 \mathrm{ml}, 14.4 \pm 4.2,4.3 \pm 2.0$. The duration of surgery, intraoperative blood loss, times of fluoroscopy intraoperatively, and postoperative pain score were as follows in group $B: 80.0 \pm 22.4 \mathrm{~min}, 121.7 \pm 25.2 \mathrm{ml}, 15.1 \pm 4.1,4.2 \pm 2.2$. And there were all no significant difference in the average duration surgery ( $p>0.05$; table 2$)$. The fracture union time in group $A$ was shorter than that in group $B \otimes 3.9 \pm 0.6 \mathrm{vs} 4.2 \pm 0.5$, $\mathrm{m} \bigotimes$,the soft tissue irritation in group $A$ was

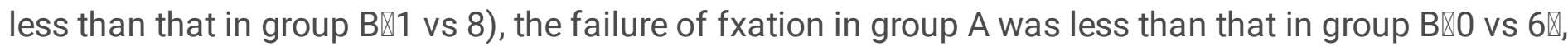
and the differences were statistically significant $(p<0.05)$. And the differences were all statistically significant $(p<0.05$; table 2$)$.

\section{Function outcome}

Broberg Morrey elbow function score: 16 excellent cases, 8 good cases, 4 fair cases and 1 poor case in group $A ; 18$ excellent cases, 7 good cases, 6 fair cases and 2 poor cases in group $B$, the difference was not statistically significant ( $p>0.05$; table 3 ).

\section{Discussion}

Olecranon fractures belong to intra-articular fracture, so malunion of olecranon fracture will influence elbow joint flexion and extension activities, thus depressing elbow joint function. Therefore, an increasing number of doctors are willing to adopt operative treatment to obtain anatomical reduction and restore the function of elbow joint to the greatest extent[6], especially for the older patients[7]. At present, there are many surgical methods for olecranon fracture, but most doctors advocated Tension band wiring (TBW) for treatment[8], which has been widely used as a method of reliable fixation for these fractures, particularly for the aged[9]. TBW avoids extensive peeling of periosteum and retains blood supply of fracture as far as possible. Secondly, the operation procedure of TBW is relatively simple. Further, it is conducive to early functional exercise[10].

However, TBW has disadvantages of Kirschner wire withdrawal, internal fixation loosening, skin irritation, and even a risk of internal fixation failure, which affect clinical efficacy[4].Elderly osteoporotic patients are more likely to lead to fixation loosening or failure [9]. So our study extends current knowledge regarding the treatment of Olecranon fractures of the aged, using the perforated Kirschner Wire to instead of standard Kirschner Wire as to providing clinical practice reference.

Our results indicate that the improved TBW technique, using the perforated K-wires, provides significantly better stability than the standard TBW technique. The results of this retrospective analysis showed that there were 6 cases of fixation failure in the standard TBW group, while no fixation failure occurred in the patients with improved TBW. The friction force between standard Kirschner wire and cortex substance is not powerful enough, so it can not limit the relative sliding of Kirschner wire in bone during elbow joint functional exercise[7]. Our team further pointed out that the bending point at the end of the Kirschner wire is not integral with the tension band, so it can not strictly restrain each other (fixation and bone) and avoid the rotation and sliding withdrawal of the Kirschner wire after operation. Due to osteoporosis of the 
aged, over time, it is easy to lead to internal fixation loosening and failure. In view of the complications above, we use the improved TBW to make up for the shortcomings of the standard TBW. The perforated Kirschner Wire is desinged with two needle holes at the tail end in order to let the tension band pass through the needle hole. Meanwhile, the tension band can be pulled in the anchor hole at the distal end of the fracture to form an integral whole and restrain each other (fixation and bone), effectively avoiding the withdrawal of the K-wire, and solving the problems of slippage or pull-out of the K-wires.

Wang[11] have made a study of biomechanics of the improved TBW, then put forward the view that the compression effect of the perforated Kirschner wire is beneficial to fracture union. The perforated hole makes the tension band and Kirschner wire form a whole, and the internal fixation obtains higher stability. During the tightening process of steel wire or steel cable, the tension can be better converted into pressure through the pulling effect of the tension band in the proximal pinhole and the distal anchor hole, which is conducive to the fracture union. In this study, we found that the fracture union time of the improved TBW group was $3.9 \pm 0.6 \mathrm{~m}$, which was less than that of the standard TBW group $(4.2 \pm 0.5 \mathrm{~m})$, and the difference was statistically significant, confirming the advantages of the improved TBW in fracture union.

In addition to the above advantages, the improved TBW also reduces the soft tissue irritation. The study of Schliemann[12] has pointed out that the withdrawal of ordinary Kirschner wire often occurs during postoperative rehabilitation exercise, which stimulates the soft tissue behind the elbow, especially the skin. So it's hard to avoid causing pain or even infection. In addition, the tail of the bent Kirschner wire adheres to the surface of the muscle tissue, leaving a protuberance of the needle tail after bending, which repeatedly rubs the surrounding skin during elbow flexion and rotation, and causes soft tissue irritation. Through using the improved TBW, soft tissue stimulation is reduced. In our practice, we identified that the needle hole of k-wire and the tension band penetrated in needle hole can be deeply buried in the deep surface of the initial part of triceps brachii, so as to reduce the stimulation of the needle tail on the soft tissue behind the elbow. The needle hole makes the tension band and Kirschner wire form a whole, which can limit the sliding and withdrawal of Kirschner wire, and further reduce the stimulation to soft tissue. According to the results of this retrospective analysis, there were 8 cases of soft tissue stimulation in the standard TBW group, which was significantly more than 1 case in the improved TBW.

In addition, the results of this study showed that there was no significant difference in operation time and blood loss between the two groups. The improved TBW is an improved design of the standard TBW, so there is no obvious difference in the reduction of olecranon fracture and the operation of Kirschner wire after reduction. Therefore, the improved TBW does not increase the operation difficulty and intraoperative blood loss. And the two kinds of internal fixation did not penetrate into the elbow joint, so they do not affect joint activity. Both the two fixation can used for early functional exercise, therefore, there was no significant difference in long-term joint function between the two groups.

\section{Conclusion}


Compared with the standard TBW, the improved TBW can not only reduce complications of olecranon fracture of the aged, but aslo promote fracture union and improve clinical curative effect without increasing operation difficulty and reducing elbow joint function. Patients with indications can adopt this treatment method.

\section{Abbreviations}

TBW means Tension Band Wire

\section{Declarations}

1. Ethics approval and consent to participate

The study was approved by the ethics committee of Rui'an people's Hospital. All clinical practices and observations were conducted in accordance with the Declaration of Helsinki. Informed consent was obtained from each patient before the study was conducted.

2. Consent for publication

I agree with it.

3. Availability of data and materials

Not applicable

4. Competing interests

The authors declare that they have no conflicts of interests to this work. We declare that we do not have any commercial or associative interest that represents a conflict of interest in connection with the work submitted.

5. Funding

Not applicable

6. Authors' contributions

Not applicable

7. Acknowledgements

Not applicable

\section{References}


1. Duckworth AD, Clement ND, Aitken SA, et al.The epidemiology of fractures of the proximal ulna. Injury. 2012;43:343-346.

2. Chen M J, Campbell S T, Finlay A K, et al. Surgical and Nonoperative Management of Olecranon Fractures in the Elderly: A Systematic Review and Meta-Analysis[J]. Journal of Orthopaedic Trauma, 2021, 35.

3. Terstappen A, Joosse P, Dijke C,et al. Tension Band Wiring Provides Excellent Patient-Reported LongTerm Results for Both Comminuted and Simple Displaced Olecranon Fractures[J]. Journal of orthopaedic trauma, 2020, 34(7):e239-e244.

4. Tarallo L , Mugnai R, Adani R, et al. Simple and comminuted displaced olecranon fractures:a clinical comparison between tension band wiring and plate fixation techniques.[J].Archives of Orthopaedic and Trauma Surgery, 2014, 134(8):1107-1114.

5. M.A. Broberg, B.F. Morrey, Results of delayed excision of the radial head after fracture, J. Bone Joint Surg. Am. 68 (1986) 669-674.

6. Double Tension Band Wiring for Treatment of Olecranon Fractures[J]. Journal of HandSurgery, 2014, 39(12):2438-2443.

7. Chalidis B, Sachinis N, Samoladas E, Dimitriou C, Pournaras J. Is tension band wiring technique the "gold standard" for the treatment of olecranon fractures? A long term functional outcome study. J Orthop Surg Res 2008;3:9.

8. Claessen F, Van D, Dijk C V, et al. Tension band wiring for simple olecranon fractures: evaluation of surgical technique[J]. Journal of Orthopaedics \& Traumatology Official Journalof the Italian Society of Orthopaedics \& Traumatology, 2017, 18(3).

9. Powell AJ, Farhan-Alanie OM, Bryceland JK, Nunn T. The treatment of olecranon fractures in adults. Musculoskelet Surg 2017;101:1-9.

10. Anglemyer A, Horvath H T, Bero L. Healthcare outcomes assessed with observational study designs compared with those assessed in randomized trials[J]. Cochrane database of systematic reviews (Online), 2014, 4(4):MR000034.

11. Wang K, Lu Y, Shen Y, et al. Where should the pins be placed to decrease the failure rateafter fixation of a Mayo IIA olecranon fracture? A biomechanical analysis[J]. Injury, 2020, 51(7).

12. Schliemann B, Raschke M J, Groene P, et al. Comparison of tension band wiring and precontoured locking compression plate fixation in Mayo type IIA olecranon fractures.[J]. Acta Orthopaedica Belgica, 2014, 80(1):106-11.

\section{Tables}


Table 1

Comparison of general data between two groups

\begin{tabular}{|lllll|}
\hline Items & $\begin{array}{l}\text { A Group } \\
(\mathbf{n}=\mathbf{2 9})\end{array}$ & $\begin{array}{l}\text { B Group } \\
(\mathbf{n}=\mathbf{3 3})\end{array}$ & F/X2 & P value \\
\hline Age (yr) & $68.0 \pm 11.4$ & $70.8 \pm 9.5$ & 1.055 & 0.300 \\
\hline Sex & & & & \\
male & 16 & 20 & 0.187 & 0.665 \\
female & 13 & 13 & & \\
\hline BMl index(kg/m2) & $26.4 \pm 5.2$ & $27.1 \pm 6.4$ & 0.468 & 0.641 \\
\hline Time from injury to operation (day) & $5.3 \pm 1.7$ & $5.1 \pm 2.0$ & -0.421 & 0.675 \\
\hline Mayo types & & & & \\
I & 11 & 13 & 0.014 & 0.906 \\
II & 18 & 20 & & \\
\hline
\end{tabular}

Table 2

Comparison of two groups of patients in clinical related indicators and complications

\begin{tabular}{|lllll|}
\hline Items & $\begin{array}{l}\text { A Group } \\
(\mathbf{n}=\mathbf{2 9})\end{array}$ & $\begin{array}{l}\text { B Group } \\
(\mathbf{n}=\mathbf{3 3})\end{array}$ & F/X2 & P value \\
\hline Surgery time (min) & $82.6 \pm 23.9$ & $80.0 \pm 22.4$ & -0.442 & 0.660 \\
\hline Intraoperative blood loss $(\mathrm{ml})$ & $119.6 \pm 30.1$ & $121.7 \pm 25.2$ & 0.299 & 0.766 \\
\hline Times of fluoroscopy intraoperatively & $14.4 \pm 4.2$ & $15.1 \pm 4.1$ & 0.663 & 0.510 \\
\hline Postoperative VAS score & $4.3 \pm 2.0$ & $4.2 \pm 2.2$ & -0.186 & 0.853 \\
\hline Fracture union time(m) & $3.9 \pm 0.6$ & $4.2 \pm 0.5$ & 2.147 & 0.036 \\
\hline Fixation failure & & & & \\
Yes & 0 & 6 & & 0.026 \\
No & 29 & 27 & & 0.029 \\
\hline Soft tissue irritation & & & & \\
Yes & 1 & 8 & & \\
\hline No & 28 & 25 & & \\
\hline
\end{tabular}


Table 3

Comparison of Broberg-Morre score between two

groups

\begin{tabular}{|lllll|}
\hline Items & $\begin{array}{l}\text { A Group } \\
(\mathbf{n}=\mathbf{2 9})\end{array}$ & $\begin{array}{l}\text { B Group } \\
(\mathbf{n}=\mathbf{3 3})\end{array}$ & $\mathrm{F} / \mathrm{Z}$ & P value \\
\cline { 1 - 3 } excellent & 16 & 18 & 0.000 & 1.000 \\
good & 8 & 7 & & \\
fair & 4 & 6 & & \\
poor & 1 & 2 & & \\
\hline
\end{tabular}

Figures
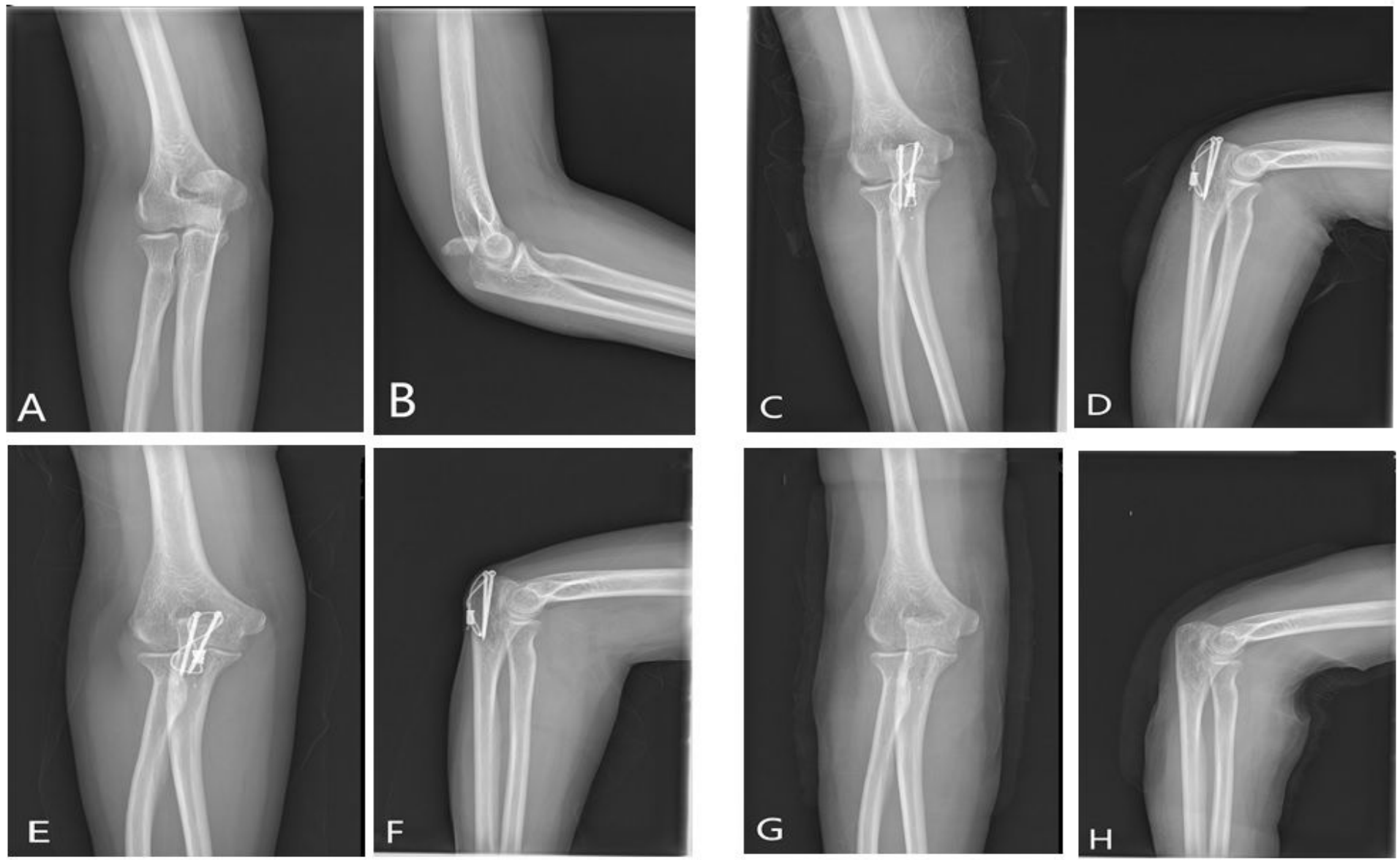

Figure 1

The improved TBW case. A B preoperative X-ray; C D the third day postoperatively; E F the third mouth postoperatively; $\mathrm{G} \mathrm{H}$ after removal of internal fixation 